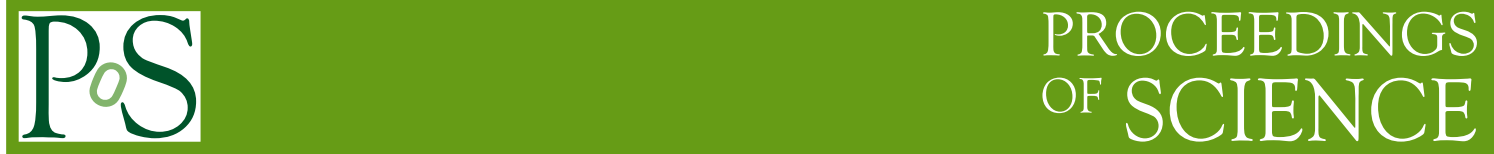

\title{
Measurement of $B_{s}^{0}$ Mixing Phase $\beta_{s}$
}

\section{Gavril Giurgiu* ${ }^{* \dagger}$}

The Johns Hopkins University

E-mail: ggiurgiuejhu.edu

The CDF and D0 experiments at the Fermilab Tevatron have recently performed the first measurements of the mixing phase $\beta_{s}^{J / \psi \phi}$ responsible for CP violation in $B_{s}^{0} \rightarrow J / \psi \phi$ decays. Each of the two experiments analysed $2.8 \mathrm{fb}^{-1}$ of data while a total of $4 \mathrm{fb}^{-1}$ have already been recorded. In the Standard Model the $\mathrm{CP}$ violation phase $\beta_{s}^{J / \psi \phi}$ is expected be very small and it is very sensitive to new physics effects. Neither of the two Tevatron experiments can provide accurate measurements of the $\mathrm{CP}$ violation phase but they can significantly constrain the allowed parameter space and search for physics beyond the Standard Model manifested as large deviation of $\beta_{s}^{J / \psi \phi}$ from zero.

2008 Physics at LHC

September 29 - 4 October 2008

Split, Croatia

${ }^{*}$ Speaker.

${ }^{\dagger}$ On behalf of the CDF and D0 collaborations. 


\section{Introduction}

Non conservation of the Charge Parity (CP) symmetry is one of the main ingredients necessary to explain the asymmetry between matter and anti-matter in the Universe. In the Standard Model (SM) of particle physics CP violation occurs due to the presence of complex parameters in the quark and neutrino mixing matrices. In particular, the Cabibbo-Kobayashi-Maskawa matrix [1] describes the transformation between weak and mass quark eigenstates. Determination of the CKM parameters has been one of the main goals in particle physics in the last decades. Within the context of the SM, CP violation effects in the quark sector measured in the kaon and neutral $B^{0}$ systems lead to the conclusion that CKM induced CP violation is not enough to explain the matter anti-matter asymmetry in the Universe. Without invoking new physics (NP), CP violation can also occur through the neutrino mixing matrix which will be studied by future long baseline neutrino experiments. In the meantime, one can search for $\mathrm{CP}$ non-conservation beyond the SM. One such possibility is to study $B_{s}^{0} \rightarrow J / \psi \phi$ decays, where $J / \psi \rightarrow \mu^{+} \mu-$ and $\phi \rightarrow K^{+} K^{-}$. In these decays $\mathrm{CP}$ violation occurs through the interference between the decay amplitudes with and without mixing. In the SM the relative phase between the decay amplitudes with and without mixing, is $\beta_{s}^{S M}=\arg \left(-V_{t s} V_{t b}^{*} / V_{c s} V_{c b}^{*}\right)$ and it is expected to be very small [2]. New physics contributions manifested in the $B_{s}^{0}$ mixing amplitude may alter this mixing phase by a quantity $\phi_{s}^{N P}$ leading to an observed mixing phase $2 \beta_{s}^{J / \psi \phi}=2 \beta_{s}^{S M}-\phi_{s}^{N P}$. Large values of the observed $\beta_{s}^{J / \psi \phi}$ would be an indication of physics beyond the SM [3].

\section{Neutral $B_{s}^{0}$ System}

A $B_{s}^{0}$ meson is a bound state composed of an anti-bottom quark $\bar{b}$ and a strange $s$ quark. The time evolution of a mixture of the $B_{s}^{0}$ and its antiparticle $\bar{B}_{s}^{0}, a(t)\left|B_{s}^{0}\right\rangle+b(t)\left|\bar{B}_{s}^{0}\right\rangle$, is given by the Schrödinger equation

$$
i \frac{d}{d t}\left(\begin{array}{l}
a \\
b
\end{array}\right)=\left(M-i \frac{\Gamma}{2}\right)\left(\begin{array}{l}
a \\
b
\end{array}\right),
$$

where $M$ and $\Gamma$ are $2 \times 2$ mass and decay matrices. The mass eigenstates $B_{L}^{0}$ and $B_{H}^{0}$ are linear combination of the flavor eigenstates $B_{s}^{0}$ and $\bar{B}_{s}^{0}$ and are obtained by diagonalizing the Hamiltonian operator. The mass eigenstates have different mass eigenvalues and the mass difference $\Delta m_{s}$ is proportional to the $B_{s}^{0}$ mixing frequency recently measured by both CDF [4] and D0 [5] experiments and found to be in good agreement with Standard Model (SM) predictions [6]. Moreover, the mass eigenstates have different decay widths difference $\Gamma_{L}$ and $\Gamma_{H}$. The average decay width is defined as $\Gamma=\left(\Gamma_{L}+\Gamma_{H}\right) / 2$ and the decay width difference is defined as $\Delta \Gamma=\Gamma_{L}-\Gamma_{H}$. The decay width difference $\Delta \Gamma=2\left|\Gamma_{12}\right| \cos \left(\phi_{S}\right)$ is sensitive to new physics effects $[6,7,8]$ that affect the phase $\phi_{s}=\arg \left(-M_{12} / \Gamma_{12}\right)$, where $\Gamma_{12}$ and $M_{12}$ are the off-diagonal elements of the mass and decay matrices. New physics can increase $\phi_{s}$, so $\Delta \Gamma$ would be smaller than the SM prediction. Since the SM phase $\phi_{S}^{S M}$ is predicted to be very small $(\approx 0.004)$ [6], in a new physics scenario with large contribution to $\phi_{s}$ one could approximate $\phi_{s}=\phi_{s}^{S M}+\phi_{s}^{N P} \approx \phi_{s}^{N P}$. If present, this new physics phase is accessible in $B_{s}^{0} \rightarrow J / \psi \phi$ decays. In these decays one can measure the CP violation phase $\beta_{s}^{J / \psi \phi}$ which is the relative phase between the direct decay amplitude and mixing followed by decay amplitude. In SM this phase is defined as $\beta_{s}^{S M}=\arg \left(-V_{t s} V_{t b}^{*} / V_{c s} V_{c b}^{*}\right)$ where $V_{i j}$ are the 
elements of the CKM quark mixing matrix. Global fits of experimental data tightly constrain the $\mathrm{CP}$ violation phase to small values in the context of $\mathrm{SM} \beta_{s}^{S M} \approx 0.02$ [9]. The presence of new physics could modify this phase by the same quantity $\phi_{s}^{N P}$ that affects the $\phi_{s}$ phase. The recent precise determination of the $B_{s}^{0}$ oscillation frequency [4] indicates that contributions of new physics to the magnitude are unlikely [8]. New physics could contribute significantly to the observed $\beta_{s}^{J / \psi \phi}$ phase $[6,7,8]$ expressed as $2 \beta_{s}^{J / \psi \phi}=2 \beta_{s}^{S M}-\phi_{s}^{N P}$. Assuming that new physics effects dominate over the SM phase, we can approximate $2 \beta_{s}^{J / \psi \phi} \approx-\phi_{s}^{N P} \approx-\phi_{s}$.

\section{Measurement of the CP Violation Phase $\beta_{s}^{J / \psi \phi}$}

The decay of the $B_{s}^{0}$ meson into $J / \psi$ and $\phi$ is a physics rich decay mode as it can be used to measure the $B_{s}^{0}$ lifetime, decay width difference $\Delta \Gamma$ and the CP violation phase $\beta_{s}^{J / \psi \phi}$. While the $B_{s}^{0}$ meson has spin 0 , the final states $J / \psi$ and $\phi$ have spin 1. Consequently, the total angular momentum in the final state can be either 0,1 or 2 . States with angular momentum 0 and 2 are $\mathrm{CP}$ even while the state with angular momentum 1 is CP odd. Angular distributions of the final muons and kaons from $J / \psi$ and $\phi$ decays can be used to separate the CP eigenstates. There are three angles that completely define the directions of the four particles in the final state. We use the angles $\vec{\rho}=\left\{\cos \theta_{T}, \phi_{T}, \cos \psi_{T}\right\}$ defined in the transversity basis introduced in Ref. [10].

An unbinned maximum likelihood fit is performed to extract the parameters of interest, $\beta_{s}^{J / \psi \phi}$ and $\Delta \Gamma$, plus additional parameters referred to as "nuisance parameters" which include the signal fraction $f_{s}$, the $B_{s}^{0}$ mass, the mean $B_{s}^{0}$ width $\Gamma \equiv\left(\Gamma_{L}+\Gamma_{H}\right) / 2=1 / \tau\left(B_{s}^{0}\right)$, the magnitudes of the polarization amplitudes in the transversity basis $\left|A_{0}\right|^{2},\left|A_{\|}\right|^{2}$, and $\left|A_{\perp}\right|^{2}$, and the strong phases $\delta_{\|} \equiv \arg \left(A_{\|} A_{0}^{*}\right)$ and $\delta_{\perp} \equiv \arg \left(A_{\perp} A_{0}^{*}\right)$. The fit uses information on the reconstructed $B_{s}^{0}$ candidate mass, the $B_{s}^{0}$ candidate proper decay time and its uncertainty, the transversity angles $\vec{\rho}$ and tag information $[4,5] \mathscr{P}$ and $\xi$, where $\mathscr{P}$ is the event-by-event correct tag probability and $\xi=\{-1,0,+1\}$ is the tag decision, in which +1 corresponds to a candidate tagged as $B_{s}^{0},-1$ to a $\bar{B}_{s}$, and 0 to an untagged candidate. The angular acceptance of the detector is calculated using simulated data and the angular analysis is validated by measuring the lifetime and polarization amplitudes in $B^{0} \rightarrow J / \psi K^{* 0}$ decays[11]. The results are consistent and competitive with most recent $B$-factory results [12]. The CDF and D0 experiments reconstruct signal samples of $\sim 3200$ and $\sim 2000$ events, respectively. The $B_{s}^{0}$ lifetime and decay width difference measured by CDF are [13] $\tau\left(B_{s}^{0}\right)=1.53 \pm 0.04$ (stat) \pm 0.01 (syst) ps and $\Delta \Gamma=0.02 \pm 0.05$ (stat) \pm 0.01 (syst) ps ${ }^{-1}$, while the corresponding D0 measurements [14] are $\tau\left(B_{s}^{0}\right)=1.52 \pm 0.05$ (stat) \pm 0.01 (syst) ps and $\Delta \Gamma=0.19 \pm 0.07$ (stat) ${ }_{-0.01}^{+0.02}$ (syst) $\mathrm{ps}^{-1}$. These are the best lifetime and decay width difference in the $B_{s}^{0}$ system to date.

An exact symmetry is present in the signal probability distribution, which is invariant under the simultaneous transformation $\left(2 \beta_{s} \rightarrow \pi-2 \beta_{s}, \Delta \Gamma \rightarrow-\Delta \Gamma, \delta_{\|} \rightarrow 2 \pi-\delta_{\|}\right.$, and $\left.\delta_{\perp} \rightarrow \pi-\delta_{\perp}\right)$. This causes the likelihood function to have two minima. Confidence regions in the $\beta_{s}^{J / \psi \phi}-\Delta \Gamma$ plane are constructed by CDF while similar confidence regions are evaluated by D0 in the $\phi_{s}-$ $\Delta \Gamma$ plane. These confidence regions are presented in Figure 1. Both results show the expected double minimum structure and they are both shifted in the same direction with respect to the SM expectation. Note that the quantities on the horizontal axes are related by $\phi_{s}=-2 \beta_{s}^{J / \psi \phi}$. The significances of the deviations are 1.8 standard deviations for CDF and 1.7 standard deviations for 

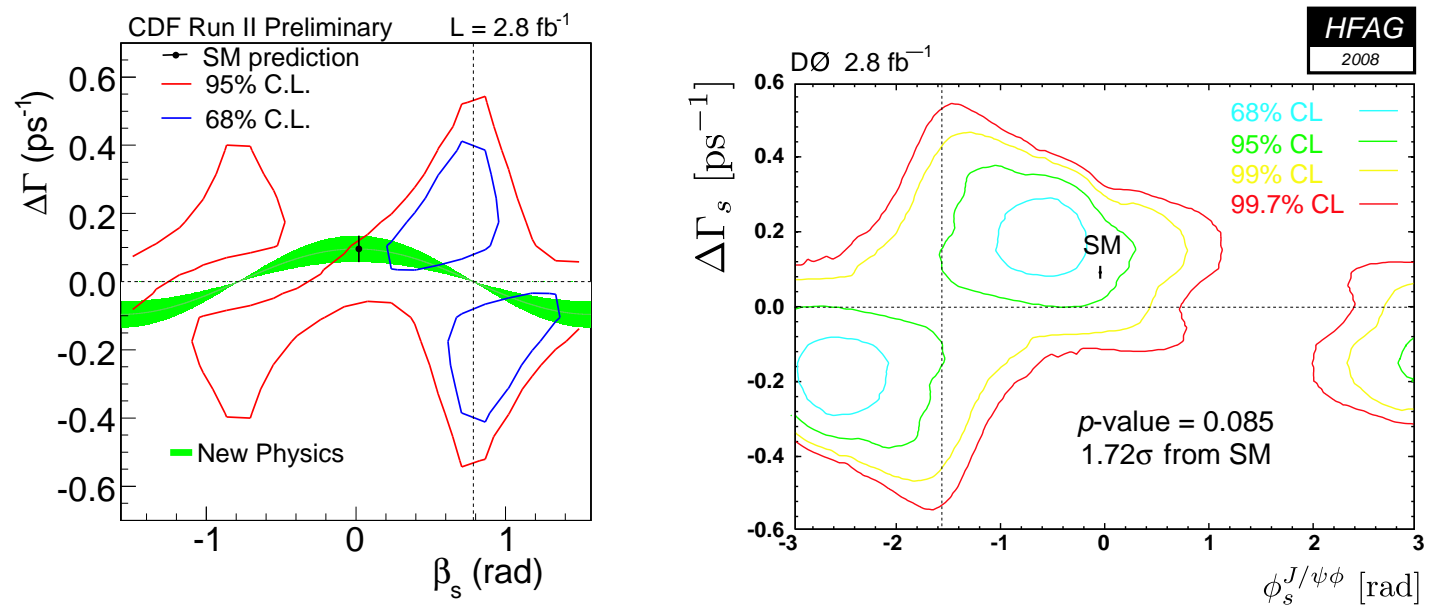

Figure 1: Confidence regions in the $\beta_{s}^{J / \psi \phi}-\Delta \Gamma$ plane from CDF (left) in the $\phi_{s}-\Delta \Gamma$ plane from D0 (right).

D0. The ambiguity between the two minima could be resolved if the strong phases $\delta_{\|}$and $\delta_{\perp}$ were known. Recent theoretical studies [15] suggest that the strong phases involved in $B_{s}^{0} \rightarrow J / \psi \phi$ decays are expected to be close to the corresponding strong phases in $B^{0} \rightarrow J / \psi K^{* 0}$. Using this information the $\mathrm{CDF}$ and $\mathrm{D} 0$ experiments show that in this hypothesis the preferred solution is the one corresponding to positive $\Delta \Gamma$.

Combinations of the CDF and D0 results have been performed $[9,16]$ and show departures between two and three standard deviations with respect to the SM prediction. These combinations include the D0 analysis with $2.8 \mathrm{fb}^{-1}$ [14] and a previous CDF result [17] that used only $1.35 \mathrm{fb}^{-1}$ of data. The combination performed in Reference [16] results in a $2.2 \sigma$ deviation of $\beta_{s}^{J / \psi \phi}$ from the SM and is shown in Figure 2. Although the combined deviation from the SM expectation is not statistically significant, the independent CDF and D0 fluctuations in the same direction are interesting to follow in the future as the analyzes will be updated using more data. By the end of the Tevatron running, samples of 6 to $8 \mathrm{fb}^{-1}$ are expected depending if the Tevatron will run until 2009 or 2010 . Figure 2 shows the CDF probability of observing a $5 \sigma$ deviation from the SM as a function of $\beta_{s}^{J / \psi \phi}$ assuming $\Delta \Gamma=0.1 \mathrm{ps}^{-1}$. The extrapolation assumes no further improvements of the analysis. However, improvements in the use of particle identification, tagging power and sample size by using additional triggers are expected from CDF while D0 will optimize the signal selection for better signal to background.

\section{Conclusions}

Both CDF and D0 experiments have performed the first measurements of CP violation in $B_{s}^{0} \rightarrow J / \psi \phi$ decays. Although the accuracy of these measurements is limited, significant regions in the $\beta_{s}^{J / \psi \phi}-\Delta \Gamma$ plane are excluded. Positive values of $\beta_{s}^{J / \psi \phi}$ are preferred by both experiments. The combined CDF and D0 measurement shows a 2.2 standard deviation departure from the SM prediction. Although not significant it is interesting to see how the $\beta_{s}^{J / \psi \phi}$ measurements evolve as more data is accumulated. 

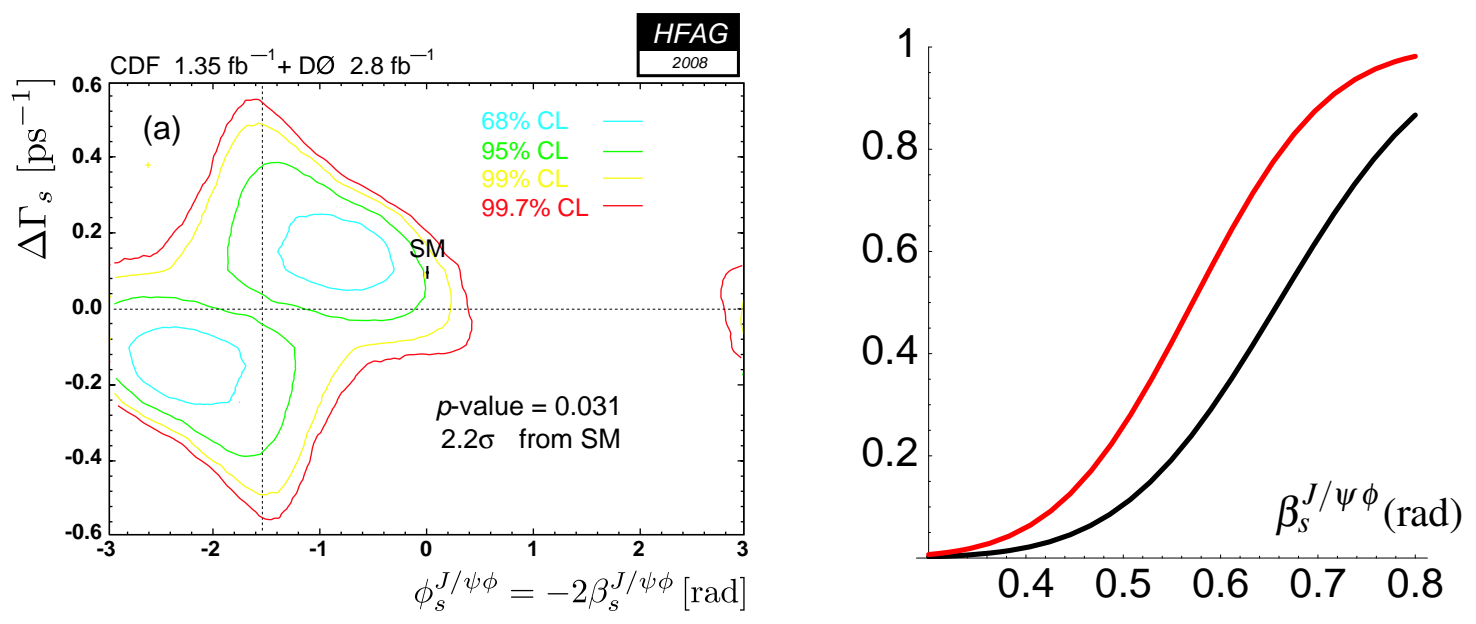

Figure 2: Confidence regions in the $\phi_{s}-\Delta \Gamma$ plane corresponding to the combined CDF and D0 datasets (left). Probability of CDF observing a $5 \sigma$ deviation from the SM prediction as a function of $\beta_{s}^{J / \psi \phi}$ assuming $6 \mathrm{fb}^{-1}$ (black line) and $8 \mathrm{fb}^{-1}$ (red line).

\section{References}

[1] N. Cabibbo, Phys. Rev. Lett. 10, 531 (1963)

M. Kobayashi and T. Maskawa, Prog. Teor. Phys. 49, 652 (1973) Phys. Rev. Lett., 51, 1945 (1983)

[2] I.I.Y. Bigi and A.I. Sanda, Nucl. Phys. B193, 85 (1981)

[3] I. Dunietz, R. Fleischer, and U. Nierste, Phys. Rev. D 63, 114015 (2001)

[4] A. Abulencia et al. (CDF Collaboration), Phys. Rev. Lett. 97, 242003 (2006)

[5] http://wWw-d0.fnal.gov/Run2Physics/WWW/results/prelim/B/B54/

[6] A. Lenz, U. Nierste, J. High Energy Phys. 06, 072 (2007)

[7] W.-S. Hou, M. Nagashima, and A. Soddu, Phys. Rev. D, 76, 016004 (2007) W.-S. Hou, M. Nagashima, and A. Soddu, Phys. Rev. Lett. 95, 141601 (2005)

[8] Z. Ligeti, M. Papucci, G.Perez, Phys. Rev. Lett. 97, 101801 (2006)

[9] J. Charles et al., Eur. Phys. J. C 41, 1 (2005), http: / / ckmfitter.in2p3.fr M. Bona et al., J. High Energy Phys. 10, 81 (2006), www . ut fit. org

[10] A. S. Dighe, I. Dunietz and R. Fleischer, Eur. Phys. J. C 6, 647 (1999).

[11] http://www-cdf.fnal.gov/physics/new/bottom/070830.blessed-BdPsiKS V. Abazov et al. (D0 Collaboration), arXiv:/0810.0037

[12] B. Aubert et al. (BaBar Collaboration), Phys. Rev. D 76, 031102(R) (2007)

R. Itoh et al. (Belle Collaboration), Phys. Rev. Lett. 95, 091601 (2005)

[13] http://www-cdf.fnal.gov/physics/new/bottom/

[14] V. Abazov et al. (D0 Collaboration), Phys. Rev. Lett. 101, 241801 (2008)

[15] M. Gronau and J.L. Rosner, Phys. Lett. B 669:321-326 (2008)

[16] E. Barberio et al. Heavy Flavor Averaging Group, 2007, http://www.slac.stanford.edu/xorg/hfag/

[17] T. Aaltonen et al. (CDF Collaboration), Phys. Rev. Lett. 100, 121802 (2008) 